\title{
The Overall Design of the Production Line Palletizing Robot
}

\author{
Bin $\mathrm{HE}^{1, \mathrm{a}}$, Hai-Nan NIU², Quan LIU'1, Yu-Qi LIU ${ }^{3}$ \\ ${ }^{1}$ Beijing Information Science and Technology University, China \\ ${ }^{2}$ School of economics and management, Changsha University of Science and Technology, China \\ ${ }^{3}$ Zhou Enlai School of Government, Nankai University, China \\ awfalw104@vip.qq.com
}

Keywords: Palletizing Manipulator, Mechanical Structure, Design.

\begin{abstract}
This study describes the production process of glass production lines; according to the production process is designed to take the line terminal board manipulator, using Pro/E software to design the mechanical structure of the three-dimensional simulation, but also gives some standard parts design and selection. Complete the control strategy of the robot.
\end{abstract}

\section{Introduction}

In the production line, palletizing robot needs to be able to quickly and accurately pick up, place and handling objects. They should request high precision, fast response, certain carrying capacity, sufficient working space and flexible freedom and the properties can be automatically positioning at any position. Palletizing robot design principles have: Comprehensive analysis of the job object of the technical requirements of the job, planning the most reasonable job process and technology, and to meet the system functional requirements and environmental conditions; Clarify the structure of the workpiece shape and material properties, positioning accuracy, crawl, mechanical characteristics during transportation, the size and quality parameters, and to determine the structure and operation of the robot control requirements; As far as possible selection of standard components, simplifying the design and manufacturing process, taking into account the general and specific nature, and can achieve a flexible conversion and programming control. The study manipulator glass production line automatic pick plate palletizing robot is suitable for large or small and medium glass production, can change the automatic handling or operation of the device operation procedures, safety requirements, professional, labor intensity large and operation frequent production monotonous occasion $^{[3]}$.

\section{Glass Palletizing System Introduced}

Glass palletizing system is a typical mechatronic system. Mechatronic Systems consists of mechanical systems, electronic information processing systems, power systems, sensor systems, actuator system, and its function as the main function, motor function, detection, control, structure function $^{[4]}$.

In the glass palletizing operation, the most common problem is the fragile glass, easy to scratch, likely to cause bodily injury of staff, in order to meet the needs of automated machinery production, palletizing robot emerged. Palletizing robot has palletizing speed, pick and place accurate, and flexible operation, reduce the labor intensity and personal injury, improved working conditions and reduce glass damage rate.

The design of three main modules, the transmission module, flip module, stacking module. Manipulator overall 3D design is shown in Figure 1.1. 


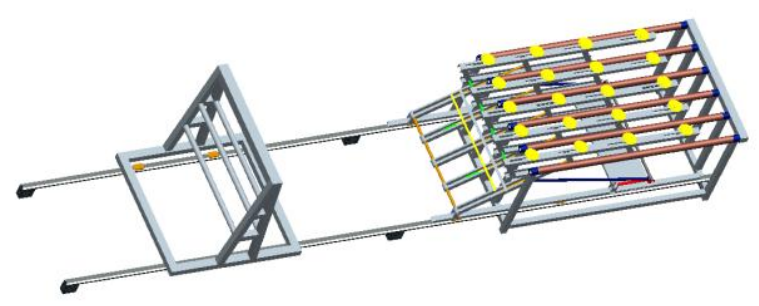

Fig.1.1 Glass Production Line Palletizing Robot 3D Design

\section{Glass Mechanical Design}

Analyzing the palletizing robot process is to determine the mechanical structure design. According to different functions, the design is divided into transmission system design, flipping system design, stacking system design; the following were separately designed for each system.

\section{Transmission System Design}

Design robot transmission system is mainly divided into stick design and transmission stent design according to the requirements of the original glass production lines; transmission roller spacing and diameter transmission line with the previous design requirements are the same, namely a known size. Its 3D design is shown in Figure 2.1. Transmission roller chain drives adoption, on one side of the roller installation.

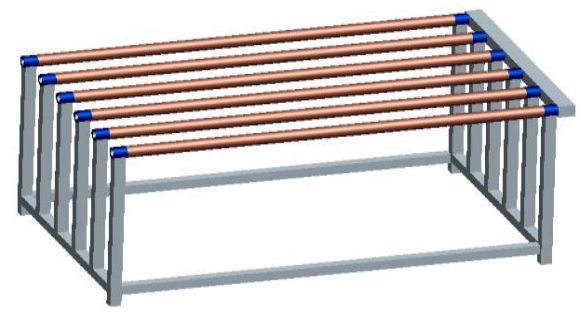

Fig.2.1 Transmission System 3D Design

\section{Flip System Design}

Flip system design is mainly divided into the sucker arm design and crank rocker mechanism design in two parts. Flip overall system diagram shown in Figure 2.2.

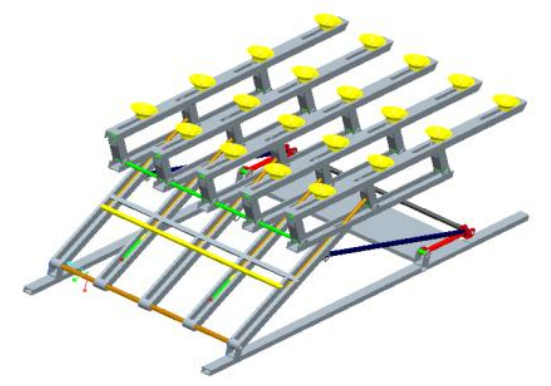

Fig.2.2 Flip Overall System Diagram

Sucker Arm Design. According to the size of the glass, the overall size of the manipulator and the distance between the single suction cup arm and the structure size are calculated. Minimum size of glass is $400 \mathrm{~mm} \times 400 \mathrm{~mm}$, Maximum size is $2000 \mathrm{~mm} \times 2000 \mathrm{~mm}$, the distance between the individual suction cup arm is d': Roller diameter $d<d$ ' $<400 \mathrm{~mm}$, Preliminary selection of the distance between a single suction cup arm $\mathrm{d}^{\prime}=300 \mathrm{~mm}$, The overall size of the manipulator is defined as the width $l_{1}=1700 \mathrm{~mm}$, length $l_{2}=2000 \mathrm{~mm}$, The number of single suction cup arm is $\mathrm{n}=$ 


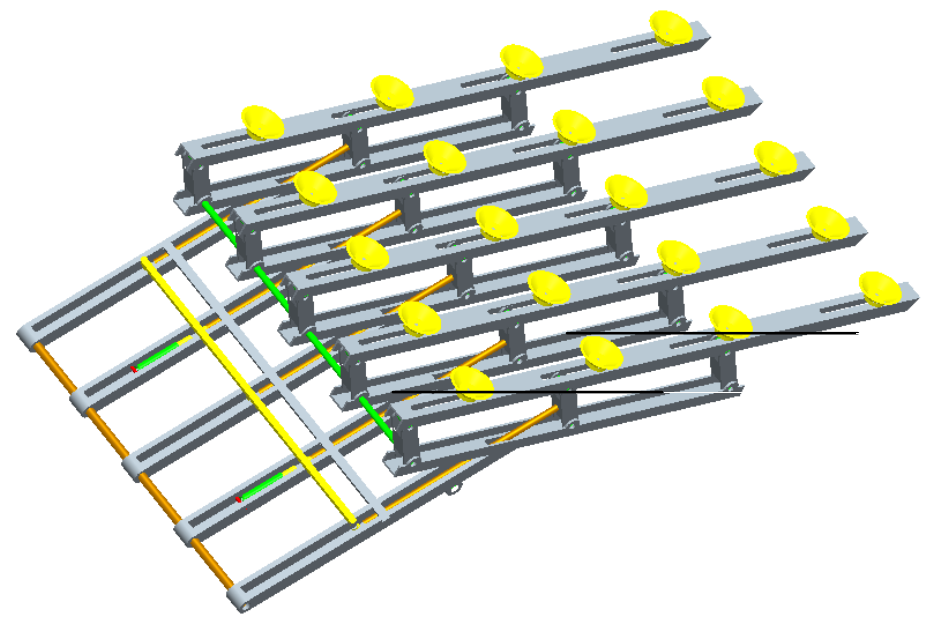

Fig.2.3 Mechanical Hand Suction Cup Arm 3D Design (1-small arm; 2-arm)

In the process of stacking glass production line, it is necessary to take a glass from the rollers down, follow the principle of taking flat level, thus lifting device selection parallelogram linkage. Vertical parallelogram linkage rod that is parallel and equal length double crank mechanism, it has a member is simple, easy manufacturing, low vice structure, high transmission accuracy, wear resistance, etc., and drive means cooperating to achieve glass pick and place, the level of mobile positioning ${ }^{[1]}$.Positioning the lifting displacement of the parallel quadrilateral mechanism $200 \mathrm{~mm}$, The angle between the crank and connecting rod as $45^{\circ}$, the length of the crank $1_{\text {crank }}=200 \times$ $\sqrt{3} \approx 350 \mathrm{~mm}$.

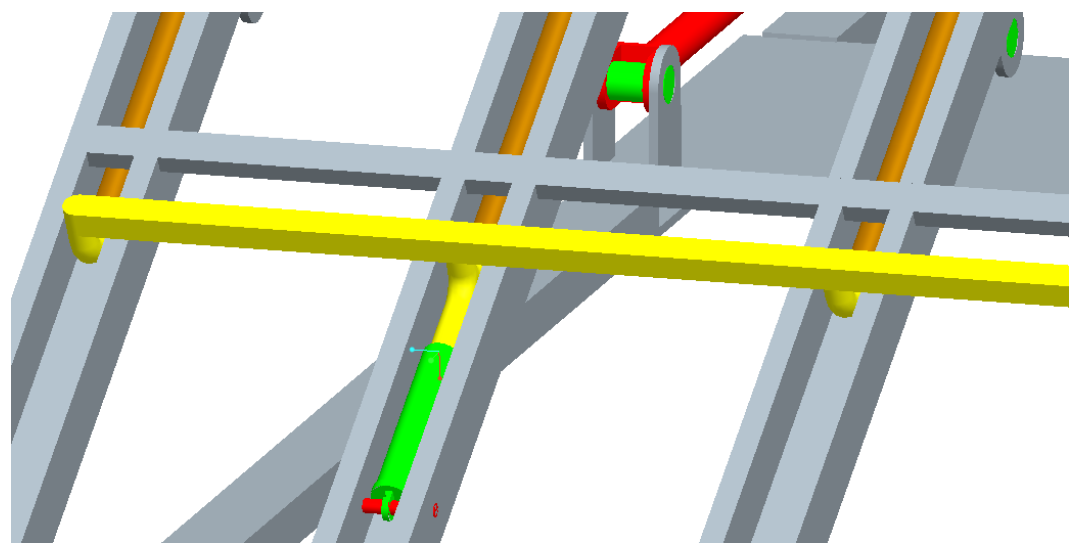

Fig.2.4 Transverse Links Enlarged View

Since the pneumatic drive system moves quickly, responsive, resistance and leakage loss is small, low cost, and therefore the drive chuck frame using pneumatic transmission mode ${ }^{[5]}$. Mounting position of the cylinder shown in Figure 2.3, the invert arm is designed to form a U-shaped groove, both to prevent the erosion of dust on the cylinder, the manufacturing process is simple, and can reduce the difficulty of installation of the cylinder. Which is installed in the U-shaped groove only two cylinders, the other U-line slots are connected with the sucker rod holder, each U-shaped groove of the cylinder rod with a horizontal rod connected to a partially enlarged diagram in Figure 2.4 shown, so not only reduces the number of cylinders, and reducing costs.

Crank Rocker Mechanism. This design will crank rocker mechanism design in the form of two interconnected, as shown in Figure 2.5, only one set of a motor-driven crank rocker mechanism, flip 
the arms on both sides of the two groups can be driven by a drive rod, both to reduce the number of motors, but also reduces the suction cup arm shaking during exercise, increasing the stability and reliability of the entire organization.

Slider-crank mechanism and the reversing mechanism rail link and form a quadrangle, there are two extreme positions of the quadrilateral form a triangle $\mathrm{ABC}$ when the crank and connecting rod collinear, when the crank and a rail line, connecting rod rail and flip arm form another triangle DBE. Flip known length of the arm and the rail base, according to the relationship between the sides of a triangle theorem, triangle law of cosines, transmission angle $\gamma_{\min } \geq 40^{\circ} \sim 50^{\circ}\left(\gamma_{\min }=40^{\circ}\right)$ Calculate the length of the crank and rocker. Take $\angle \mathrm{ABC}=110^{\circ}, \angle \mathrm{BDE}=30^{\circ}, l_{A B}=1000 \mathrm{~mm}$. By the law of cosines can be obtained:

$$
\begin{aligned}
& \cos \angle A B C=\frac{l_{A B}{ }^{2}+l_{B C}{ }^{2}-l_{A C}{ }^{2}}{2 l_{A B} l_{B C}} \\
& \cos \angle B D E=\frac{l_{B D}{ }^{2}+l_{D E}{ }^{2}-l_{A C}{ }^{2}}{2 l_{B D} l_{D E}}
\end{aligned}
$$

By the sides of a triangle relation theorem can be obtained:

$$
\begin{aligned}
& l_{A B}+l_{B C}>l_{A C} \\
& l_{B D}+l_{D E}>l_{B E} \\
& l_{A B}=l_{B D}=1000 \mathrm{~mm}, l_{B C}=2000 \mathrm{~mm}, l_{A C}=l_{B E}+l_{C E}: l_{D E}=1800 \mathrm{~mm}, l_{C E}=1000 \mathrm{~mm} .
\end{aligned}
$$

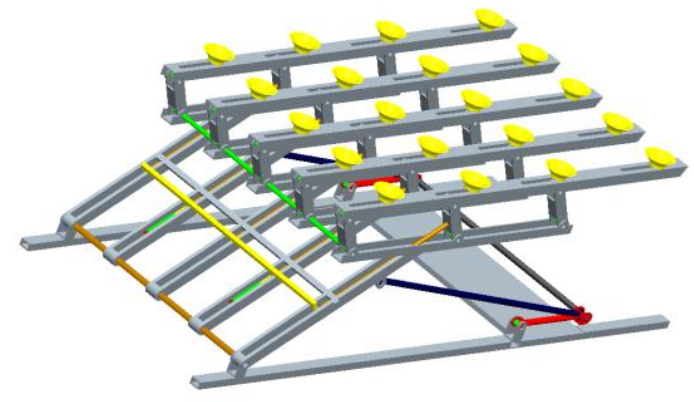

Fig.2.5 Crank Rocker Mechanism 3D Design

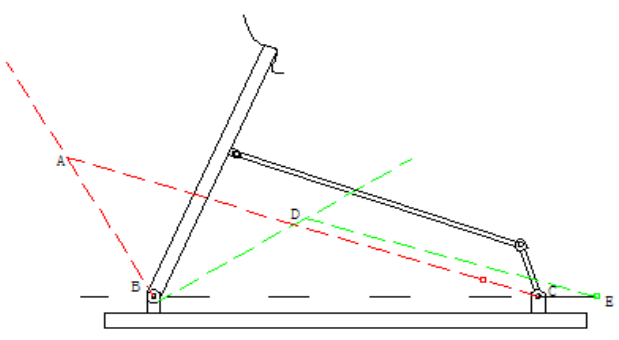

Fig.2.6 Single Crank Rocker Mechanism Trajectory Design

\section{Stacking System Design}

A stacked shaped stent using the stent according to the maximum size of the glass $2000 \times$ $2000 \mathrm{~mm}$, the minimum size of $400 \times 400 \mathrm{~mm}$, the height of the stent may be designed to $2 \mathrm{~mm}$, a 
width of $2 \mathrm{~mm}$, the angle between the bracket and the horizontal arm can be set flip angle, i.e. $\theta=$ $180^{\circ}-110^{\circ}=70^{\circ}$. As shown in Figure 2.7, in order to place the various sizes of glass, the intermediate support structure made of beams, the minimum size may be height glass transom with the bottom surface of the stent between to be, that is, $h=400 \mathrm{~mm}$. The design of the stent is designed to be slidable along the guide rail form, which can effectively prevent the phenomenon of the glass roll stacking process because of varying quality caused by the mounting brackets, increase the stability of the stent and the stent can slide along the guide rail, flexible Convenience.

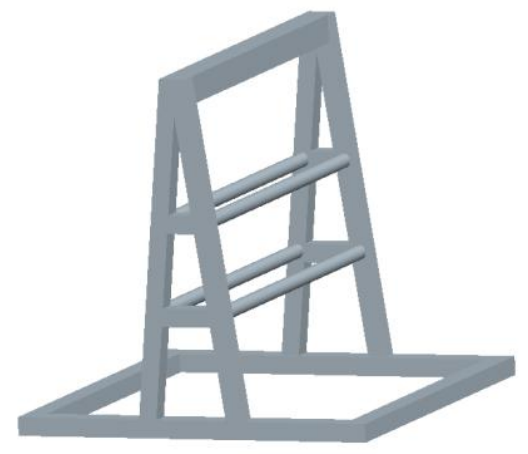

Fig.2.7 Stacked Glass Holder

\section{Summary}

This chapter analyzes the process of palletizing robot designed 3D map each section. This chapter focuses on the invert arm mechanism, sucker arm mechanism, rocker mechanism, stacking bracket Figure 3D modeling, and the use of Pro / E design three-dimensional model, and derive two-dimensional design drawings. Although the design is not the best, but after the redesign and improvement of a certain model, it is of great significance for improving the efficiency of glass production line palletizing.

\section{References}

[1] Wang Zhong.Its application to expand the parallelogram mechanism [J]. Mechanical Design.2005, (12):504 530.

[2] Michal Šingliar. Solar energy using for hydrogen production [J]. Petroleum and Coal. 2007.

[3] Feng Gui-ming, Zhao Hai-xue. On the ultra-white rolled glass photovoltaic common molding defects [J] .Glass. 2011(10):21 to 23.

[4] Tian Da-wei. Research on mechatronics [J]. Online education network Herald. 2012(45): 96 99.

[5] Xie Xue-mei. Experimental study of glass defects detection system [D]. Qingdao: China Ocean University. 2008.05.

[6] Zhou Yang. Glass quality online detection algorithm research and system implementation [D]. Wuhan: Huazhong University of Science and Technology. 2006.04. 